\title{
La eficacia de la policía estatal en México
}

\author{
Luis Felipe Llanos, Luis Arturo Rivas-Tovar, ${ }^{* *}$ Fernando Lambarry,* \\ Mara Maricela Trujillo****
}

Perfiles Latinoamericanos, 26(52) | 2018

DOI: $10.18504 / \mathrm{pl} 2652-015-2018$

Recibido: 8 de diciembre de 2015

Aceptado: 16 de junio de 2017

\section{Resumen}

El objetivo de esta investigación consiste en estudiar la eficacia de la policía estatal mexicana por medio del impacto de su diseño organizacional y el desarrollo del factor humano. Para ello se ha utilizado como método el análisis causal multivariado. Los resultados indican que la especialización de las funciones de los cuerpos policiacos, la falta de agentes, la infiltración del crimen, la ausencia de lealtad y la negligencia, son variables estadísticamente significativas en su eficacia, por lo que se propone un índice considerando aspectos como el número de homicidios, autos asegurados robados, la percepción ciudadana de inseguridad y la calificación ciudadana de la actuación de la policía, lo que podría ser útil para mejorar la eficacia de la policía.

\begin{abstract}
The objective of the investigation is to study the effectiveness of Mexican state police through the impact of organizational design and the development of the human factor. For this, the method was based on multivariate causal analysis. The results indicate that the specialization of police functions, lack of agents, infiltration of crime, lack of loyalty and negligence are statistically significant in their effectiveness. Therefore, an index is proposed, considering the number of homicides, stolen auto insured, citizen perception of insecurity and citizen rating of police action, which can serve to improve the effectiveness of the police.
\end{abstract}

Palabras clave: eficacia de la policía mexicana, diseño organizacional, desarrollo del factor humano, policía estatal, México.

Keywords: Effectiveness of the Mexican police, organizational development, human development, state police, Mexico.

* Doctor en Ciencias Administrativas por el Instituto Politécnico Nacional (México). Universidad Anáhuac México | luis.llanos@anahuac.mx

** Doctor en Estudios Europeos por el Instituto Ortega y Gasset (España). Escuela Superior de Comercio y Administración, Unidad Santo Tomás del Instituto Politécnico Nacional | larivas33@hotmail.com

*** Doctor en Ciencias Administrativas por el Instituto Politécnico Nacional (México). Escuela Superior de Comercio y Administración, Unidad Santo Tomás del Instituto Politécnico Nacional | flambarry@ ipn.mx

**** Doctor en Ciencias Administrativas por el Instituto Politécnico Nacional (México). Escuela Superior de Comercio y Administración, Unidad Santo Tomás del Instituto Politécnico Nacional | mtrujillof@ipn.mx 


\section{Introducción}

- 2 as corporaciones de policía en México no han resuelto el problema de cómo abatir la inseguridad que perciben los ciudadanos y de cómo mejorar su eficacia en contra de los criminales. La población requiere del gobierno una respuesta para obtener una mayor seguridad en todos los niveles. Este ya es un problema de salud pública (Benítez, 2010). Ante ello, Alpert (1994), Mastrofski (1999), Purpura (2001), Rico \& Chinchilla (2002), National Research Council (2004) y Salamanca (2004) proponen dos dimensiones para medir la eficacia de las policías, la primera es objetiva y remite al volumen de delitos; la segunda es subjetiva y se relaciona con la sensación de inseguridad de la ciudadanía.

En México, los indicadores oficiales de la percepción ciudadana acerca de la inseguridad y los de confianza respecto de las corporaciones de policía han disminuido en los últimos años: 1) El Instituto Nacional de Estadística Geografía e Informática (INEGI) realiza desde 2010 la Encuesta Nacional de Victimización y Percepción de la Seguridad Pública (ENVIPE). En esta encuesta los ciudadanos califican de deficiente el desempeño de las policías estatales. Para 2012, solo el $7.7 \%$ de la población las considera como muy efectivas, un porcentaje por debajo de la calificación del ejército, la armada y las policías federales (INegi, 2012a). 2) Desde 2009, el INEgi mide el Índice de Percepción de Seguridad Pública con un valor inicial de 100 puntos, el cual se modifica mensualmente dependiendo de los resultados de la medición de la seguridad personal, actual y esperada de los habitantes que arroja la encuesta ENVIPE. Desde su creación este índice observa una tendencia prácticamente horizontal. A junio de 2013 tiene un valor de 107 puntos (INEGI, 2012b).

En 2006, el secretario de Seguridad Pública en México planteó que la coordinación de las corporaciones es uno de los obstáculos para combatir la criminalidad, ya que con 1661 agrupaciones no se han podido mejorar los índices de seguridad (García-Luna, 2006).

La falta de precisión en la eficacia de la administración de las organizaciones de policías en México se debe posiblemente a un fenómeno multicasuístico, por lo que el objetivo de esta investigación es comprobar si existen variables del diseño organizacional y otras del desarrollo del factor humano dentro de la policía estatal preventiva mexicana que impacten en su eficacia. 


\section{Base teórica}

Diseño organizacional y desarrollo del factor humano

Las definiciones conceptuales de diseño organizacional y desarrollo del factor humano localizadas en la literatura, así como los mejores indicadores para sus mediciones se reseñan enseguida.

El diseńo organizacional es el proceso por el cual una organización crea y modifica su estructura de puestos y sus redes de decisión, de información y de sus sistemas de gobernabilidad, con el fin de cumplir con su estrategia planteada (Cook \& Hunsaker, 2001; Daft, 2015; Gibson, Ivancevich, Konopaske \& Donnelly, 2011; Griffin, Phillips \& Gully, 2017; Nelson \& Quick, 2013).

La estructura de puestos se puede clasificar en cuatro grandes dimensiones: i) el diseño de puestos, que incluye elementos como los objetivos y retos principales, las funciones y atribuciones, el perfil de los ocupantes, el sistema de remuneraciones y la carrera de avance (Abid, Sarwar, Imran, Jabbar \& Hannan, 2013; Cook \& Hunsaker, 2001; Fried \& Ferris, 1987); ii) la dimensión horizontal, la cual mide el número de departamentos especializados de una organización (Gibson, Ivancevich, Konopaske \& Donnelly, 2011; Nelson \& Quick, 2013); iii) la dimensión vertical, que mide la altura de las organizaciones, desde el primer puesto directivo, hasta el último puesto operativo (Cook \& Hunsaker, 2001; Griffin, Phillips \& Gully, 2017), y iv) la dimensión espacial, la cual mide la dispersión geográfica de la organización (Cook \& Hunsaker, 2001; Nelson \& Quick, 2013). El grado de complejidad de una organización debe estar relacionado con la complejidad de su entorno, tanto en número de actividades y unidades, como de sistemas (Lawrence \& Lorsch, 1986).

Las redes de decisión, información y de los sistemas de gobernabilidad se miden usando varias alternativas: i) la gobernabilidad, con base en la autonomía o concentración de la toma de decisiones (Gibson, Ivancevich, Konopaske \& Donnelly, 2011; Mintzberg, 1979); ii) la formalización, con el grado de regulación o formalización de procedimientos oficiales (Daft, 2015; Nelson \& Quick, 2013), iii) la centralización de la información, con base en su concentración en las altas esferas (Gibson, Ivancevich, Konopaske \& Donnelly, 2011; Mintzberg, 1979).

De acuerdo a Woodward (1958), las variables contextuales que influyen en el diseño organizacional y determinan el éxito de la estrategia de una organización y principales indicadores son: $i$ ) el tamaño, que se mide por el número de personal permanente (Gooding \& Wagner III, 1985); ii) la tecnología, es decir, las herramientas, técnicas y sistemas que utiliza la organización para sus funciones, y se 
mide con el grado de interacción de los elementos tecnológicos con el proceso de trabajo (Thompson, 1967; Tsai \& Huang, 2012), y iii) el entorno, que se mide con el número de condiciones externas a una organización que puedan afectar de manera importante sus resultados (Endrikat, Guenther \& Hoppe, 2014; Liu, Guo \& Chi, 2015).

El desarrollo del factor humano se centra en las prácticas de las organizaciones para gestionar el conocimiento y principalmente en mejorar las competencias de sus colaboradores para que desempeñen las funciones de modo confiable, a fin de que puedan progresar en sus carreras profesionales (Belamaric, Arrastía \& Cañizares, 2001; Castillo, 2012; Davenport, 2000; Hellriegel, Jackson \& Slocum, 2007). La diferencia entre capacitar y desarrollar se enfoca en el plazo de los objetivos. El primer proceso busca transmitir a los empleados los conocimientos esenciales de corto plazo para que hagan más eficiente y confiable su trabajo (Chiavenato, 2000); mientras que el segundo pretende perfeccionar a la persona, en sus valores, creencias, capacidad de aprendizaje y liderazgo, para fortalecer su crecimiento profesional y pueda alcanzar puestos de mayor complejidad (Hellriegel, Jackson \& Slocum, 2007).

Los indicadores de la capacitación y desarrollo se concentran en dos aspectos: constatar si se produjo un cambio en las conductas, y si estas se vieron impactadas en los resultados de la organización (Chiavenato, 2000; Gooding \& Wagner III, 1985; Hellriegel, Jackson \& Slocum, 2007).

\section{La medición de la eficacia de la policía}

La complejidad de evaluar la eficacia del trabajo policial se debe a que este se desenvuelve en un ambiente caótico de violencia e inseguridad, el cual no se ha considerado como un eje para medir la actuación de las instituciones de seguridad. Además, la información útil para medir su gestión depende de la propia actividad de la policía, esto es, que opera en un campo de acción donde se carece de precisión en los datos, independientemente de que estos se generen en los organismos oficiales, debido a que, en lo general, los afectados no denuncian los delitos (Cano, 2003; Cruz, Maganda, Hernández \& González, 2015).

Aunque una tendencia, bajo los supuestos de la teoría de la contingencia, es que la cultura es un predictor de la eficiencia organizacional (Vallejo, 2008), la cultura organizacional de la policía es particular a ella y diferente a otras instituciones (Crank \& Crank, 2014), lo que ha hecho que se la conceptualice diversa y controversialmente en su delimitación (Cockcroft, 2013; Paoline \& Terrill, 2014). De esta forma se ha asumido que la cultura organizacional de la policía es resultado de la influencia del entorno y de un conjunto complejo de determi- 
nantes situacionales (Mastrofski, 2004), por lo que dar una definición puede ser $\tan$ intrincado como poco definitivo (O’Neill, Marks \& Singh, 2007).

Por otra parte, la medición de la eficacia ha utilizado distintos modelos: el gubernativo (Savelsberg, 1994); el profesional, forjado en la primera mitad del siglo xx por Vollmeren 1936 (Loubet Del Bayle, 2012); más recientemente, el Intelligence-Led Policing (Newburn, 2007; Tilley, 2008), el Problema-Oriented Policing (Bertaccini, 2011; Tyler, 2011), el Broken Windows Policing (Newburn, 2007), y el de la policía de aseguramiento de barrio (Bertaccini, 2011; Newburn, 2007). Todos han hecho aportaciones relevantes con resultados desiguales, en tanto que la policía de barrio se ha estudiado con profundidad en Reino Unido.

Es por ello que las investigaciones más recientes sobre la eficacia de los resultados de las organizaciones de policía, más que centrarse en al análisis de su cultura, se orientan a temas organizacionales y de factor humano. Y no existe claridad sobre su medición: los investigadores han expresado opiniones contrarias de lo que constituye un buen desempeño, básicamente porque comporta una complejidad intrínseca de su medición (Alpert, 1994).

Algunos exponen que la medición de la delincuencia es universalmente aceptada como uno de los principales indicadores para estimar el desempeño policial usando indicadores como el volumen de criminalidad, los actos de incivilidad, el esclarecimiento de delitos y el volumen del arresto de sospechosos vinculado a la recepción de denuncias (Medina, 2011; Newburn, 2007; Rico \& Chinchilla, 2002; Roberts, 2006; Salamanca, 2004; Silverman, 2002; Tilley, 2008; Wilson, 2005).

Dado el subregistro significativo de los delitos y que los ciudadanos no los denuncian, las llamadas cifras negras, ${ }^{1}$ algunos investigadores proponen usar el número de homicidios o el robo de automóviles asegurados como indicadores confiables y objetivos para evaluar la eficacia de la policía y, por ende, la seguridad del país, debido en particular a que este tipo de delitos suelen denunciarse siempre (Decker \& Pyrooz, 2010; ICESI, 2010a; National Research Council, 2004). Un segundo grupo de investigadores propone usar indicadores de calificación ciudadana sobre el desempeño de la policía y la percepción de inseguridad (Amadi, 2014; Mastrofsky, 1999; Purpura, 2001); a pesar de las críticas a este tipo de evaluación social — por su subjetividad y sesgo — es ampliamente utilizada sobre todo en el medio periodístico (Heath, 1984; Oliveira-Muniz \& Proença, 2007).

1 Cifra negra es la diferencia entre el número de delitos registrados en las estadísticas oficiales y el de delitos realmente cometidos. Este error es recurrente a escala internacional. Para subsanarlo se recurre a encuestas ciudadanas. En 2011, la Encuesta Nacional de Victimización y Percepción de la Seguridad Pública estimó la cifra negra para México en 91.6\% (INEGI, 2012c). 
El Instituto Ciudadano de Estudios sobre la Inseguridad, A. C. (ICESI) sugiere que para la medición de la eficiencia se deben usar indicadores objetivos y subjetivos, y propone tres básicos: la victimización, la percepción de la inseguridad, y la criminalidad registrada o cifra oficial (ICESI, 2016b).

Con una perspectiva de diseño organizacional, Wells \& Falcone (2005), a partir del estudio de una base de datos de más de tres mil agencias de policías locales, establecieron que hay grandes divergencias entre los organismos policiacos de diferentes tamańos y ubicaciones, y que las tareas de implementación de una policía con una orientación a procesos vs. otra con orientación a la comunidad pueden variar ampliamente, dependiendo de estos factores. Wilson (2005), por su parte, determinó por medio del estudio y análisis de fuentes oficiales de 401 organizaciones de policía, que el factor más influyente en la eficiencia de la ejecución de la policía resulta ser su ubicación; mientras que la relevancia del pago de incentivos aunque influye positivamente es más débil.

Con la perspectiva de desarrollo del factor humano, Rico \& Chinchilla (2002), mediante el estudio de la seguridad ciudadana y la reforma policial, la justicia, la policía y la seguridad en América Latina, proponen el grado de competencia de los agentes en sus actividades como índice de eficacia de una organización de policía. Wasserman \& Moore (1988), con base en las experiencias del Estado Mayor de la Casa Blanca, y las investigaciones en materia de justicia penal y de gestión, establecieron que con el solo hecho de declarar los valores de una corporación de policía explícitamente, se encamina esta hacia la excelencia de su servicio.

Oliveira-Muniz \& Proença (2007), al estudiar los métodos de las policías para combatir el crimen en Brasil, determinaron que las organizaciones deben enfocar su capacitación en el uso de la fuerza, sobre todo porque la actuación de cada policía depende de su poder discrecional en la calle para realizar su trabajo y de su fuerza de reacción; por lo que requieren una sólida formación para desarrollar su "buen juicio". Mastrofski (2007), Purpura (2001), Salamanca (2004) y Servera-Muntaner (2006) proponen que los policías requieren conocer y entender los valores éticos y las formas aceptadas para el uso de la fuerza, por lo que determinaron tres variables que influyen en los niveles de capacitación de sus elementos: la antigüedad en la organización, la movilidad interna y, de forma negativa, la rotación del empleo (Medina, 2011; Savelsberg, 1994; Wells \& Falcone, 2005).

Finalmente, en las últimas cuatro décadas los departamentos de policía se han convertido en equipos cada vez más profesionales para hacerse cargo de los requerimientos de su comunidad, por lo que han optado por la participación de la ciudadanía en sus procesos de entrenamiento (Salamanca, 2004; Savelsberg, 1994; Shane, 2007; Wasserman \& Moore, 1988; Wilson, 2005). 
Por otra parte, la formación por sí sola no mejora una organización de policía, se requiere además acompañarla de un programa sistemático de evaluación (Mastrofski, 2007; Medina, 2011; Newburn, 2007; Tilley, 2008; Tyler \& Huo, 2002).

Un punto importante es el hecho de que en México por décadas han existido diversos tipos de policías: la municipal, la estatal y la federal, con funciones meramente preventivas; quien auxiliaba en la investigación y persecución de delitos al Ministerio Público era la policía judicial. Por ello se implementó un nuevo modelo de policía a nivel nacional, el cual, mediante la restructuración de las corporaciones policiales, se esperaba que fortaleciera la construcción de capacidades de operación y privilegiara el desarrollo de sus miembros (Secretaría de Seguridad Pública Federal, 2010).

En la tabla 1 se presenta el análisis de las dimensiones identificadas en el fenómeno multicasuístico discutido hasta aquí.

Tabla 1. Fenómeno multicasuístico respecto a eficacia de la policía

\begin{tabular}{|c|c|c|c|c|c|c|c|c|c|}
\hline Dimensiones & 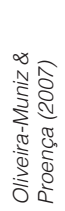 & 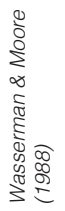 & 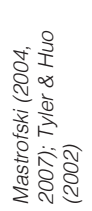 & 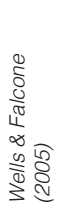 & 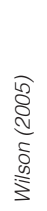 & 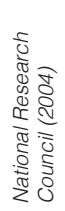 & 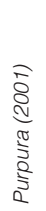 & 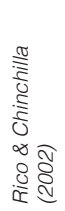 & 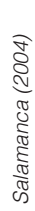 \\
\hline Confiabilidad & & & $x$ & & & $x$ & $x$ & $x$ & $x$ \\
\hline Crecimiento profesional & $x$ & & $x$ & & & & & $x$ & \\
\hline Distribución geográfica & & $x$ & $x$ & $x$ & $x$ & & & $x$ & \\
\hline Equipamiento y equipo & & & $x$ & & & $x$ & & & \\
\hline $\begin{array}{l}\text { Especialización de las } \\
\text { funciones }\end{array}$ & & & & $x$ & $\mathrm{x}$ & & $x$ & $x$ & $x$ \\
\hline $\begin{array}{l}\text { Estandarización de los } \\
\text { procesos }\end{array}$ & $x$ & & $x$ & $\mathrm{x}$ & $x$ & & & $x$ & \\
\hline Evaluación ciudadana & & & $x$ & & & & $x$ & $x$ & $x$ \\
\hline $\begin{array}{l}\text { Capacitación de recursos } \\
\text { humanos }\end{array}$ & $x$ & $x$ & $x$ & & $x$ & $x$ & $x$ & & $x$ \\
\hline Gobernabilidad & $x$ & $x$ & $x$ & & $x$ & & $x$ & & \\
\hline Cantidad de jerarquías & & & & $x$ & $x$ & $x$ & & & \\
\hline Oportunidad de respuesta & $x$ & $x$ & $x$ & & & & & $x$ & \\
\hline Presupuesto & & & $x$ & & & $x$ & $x$ & $x$ & \\
\hline $\begin{array}{l}\text { Respeto a los derechos } \\
\text { humanos }\end{array}$ & & $x$ & $x$ & & & & $\mathrm{x}$ & $x$ & $x$ \\
\hline Sistemas de información & & & $x$ & & $x$ & $x$ & $x$ & & \\
\hline Remuneraciones & & & & $x$ & $x$ & & & & \\
\hline Tamaño de la organización & & & $x$ & $x$ & $\mathrm{x}$ & & & & $x$ \\
\hline Valores & $x$ & $x$ & & & & $x$ & $x$ & & $x$ \\
\hline Volumen de la delincuencia & $\mathrm{x}$ & $\mathrm{x}$ & $x$ & $\mathrm{x}$ & $\mathrm{x}$ & $x$ & & $\mathrm{x}$ & $\mathrm{x}$ \\
\hline
\end{tabular}

Fuente: Elaboración propia. 
Por la frecuencia de los temas identificados en la literatura especializada, se puede argumentar que para medir la eficacia de los cuerpos de policía existen numerosas dimensiones que deben considerarse.

\section{Método de investigación}

Esta investigación ha optado por un enfoque cuantitativo causal multivariado a fin de probar si la eficacia de la policía estatal preventiva está significativamente explicada por su diseńo organizacional y el desarrollo del factor humano. Se ha usado solo información oficial disponible de las 32 corporaciones estatales de policía, que corresponden a la misma cantidad de entidades federativas de la república mexicana. El diseño es no experimental debido a sus características de temporalidad y ser transaccional (Hernández-Sampieri, Fernández \& Baptista, 2006; Rivas, 2006).

La hipótesis de investigación se ha planteado en los términos siguientes: H0: El diseño organizacional y el desarrollo del factor humano están positivamente relacionados con la eficacia de las policías.

La variable dependiente Eficacia de la Policía (EF), se la ha definido como un índice compuesto de cuatro indicadores, dos de naturaleza objetiva: a) robo de automóviles asegurados, y b) homicidios por habitante; y dos subjetivos: c) población que califica como poco o nada efectivo el desempeño de la policía estatal, y d) población que percibe sentirse insegura en su estado. Todos son indicadores con la misma ponderación. Para reducir el sesgo a favor de alguno, los datos de cada uno se han relativizado dividiendo entre su promedio respectivo (Muñiz, 2000).

Una limitante de los indicadores seleccionados se presenta si el delito de robo $\mathrm{u}$ homicidio se realiza en una entidad federativa pero se registra en otra por la cercanía del hecho con alguna de sus colindancias geográficas; sin embargo, tanto la literatura especializada (Decker \& Pyrooz, 2010; ICESI, 2010a; National Research Council, 2004), como el Beter Life Index de la Organización para la Cooperación y el Desarrollo Económicos (OCDE) (2017) identifican estos indicadores como los más confiables en términos objetivos.

Para una primera discriminación de las variables independientes, de la información generada por los organismos oficiales se utilizaron el contexto y las características de los sujetos de estudio, y a partir de esto se definieron los criterios de identificación y selección que se enumeran enseguida (Maella, 2010):

1. Que hayan sido utilizadas en investigaciones académicas sobre los modelos de las organizaciones de policías entre 1978 y 2008. 
2. Que existan datos que provengan de organismos oficiales registrados entre enero de 2011 y diciembre de 2012.

3. Que la información sea pública con la reserva y confidencialidad de la Ley Federal de Transparencia y Acceso a la Información Pública Gubernamental.

4. Que si los datos provienen de una fuente primaria de cuestionarios, estos tengan al menos cuatro opciones de respuesta, a fin de aumentar la probabilidad de que la distribución de los datos consecuente se acerque a la normalidad (Arias, 2008).

5. Que sus coeficientes de correlación tengan una consistencia teórica respecto de los cuatro indicadores de la variable dependientes. Esta regla, de acuerdo con Gujarati (1993: p. 338) establece que: "Un modelo puede no ser bueno, a pesar de que se obtenga un $\mathrm{R}^{2}$ alto, si uno o más de sus coeficientes estimados tienen los signos equivocados, por lo que se debiera cuestionar mucho el resultado obtenido". El mismo autor agrega que uno de los atributos de un buen modelo es su consistencia teórica. $\mathrm{Al}$ respecto expone que hay que: "mantener un modelo tan simple como sea posible" (p. 337). Por su parte, Kennedy (2002: p. 572) recomienda: "Usar el sentido común". Más adelante, Kennedy (2005) observa que el signo de una variable clave se puede utilizar como una prueba estadística de la especificación general del modelo que se está estimando, por lo que si el signo está significativamente equivocado, se podría eventualmente rechazar, concluye diciendo que "esto debería iniciar una investigación importante por el investigador para determinar la razón de este rechazo" (p. 215). En este sentido se establece que se deben omitir del modelo aquellas variables de fuentes oficiales que presenten dos o más coeficientes de correlación con signo contrario en relación con cada uno de los cuatro indicadores de la variable dependiente.

En cuanto a los posibles datos atípicos que se identifique en las variables seleccionadas, se aplicará el método de truncar aquellos que estén alejados más de tres desviaciones estándar de su media (Arias, 2008), a fin de eliminar el efecto negativo que pudieran causar en el proceso de mínimos cuadrados (Johnston, 2000; Osborne \& Overbay, 2004). De igual modo, por el tamaño del universo a analizar no es recomendable desechar variables que pudieran presentar datos faltantes (McKnight, McKnight, Sidani \& Figueredo, 2007), por lo que, de surgir esto en alguna variable dependiente, se recurrirá el método de Frane (1976) consistente en la estimación de los faltantes mediante una ecuación de regresión lineal, calculada con base en el vector de otra variable dependiente que manifieste la mejor correlación.

Para examinar y determinar las relaciones significativas entre las variables independientes y la variable dependiente observada, en otro aspecto, el método 
aplicado fue el del modelo de análisis de regresión múltiple, que a través de la R-sq(adj) identifica las relaciones significativas de variables (Johnston, 1972; Salvatore, 1994; Gujarati, 1993).

\section{Resultados}

Para seleccionar las posibles variables, se identificaron fuentes de información oficial con datos de indicadores objetivos y subjetivos relacionados con la eficacia de las 32 policías estatales en México: Comisión Nacional de Seguros y Fianzas (CNSF), Instituto Nacional de Estadística y Geografía (INEGI), Secretariado Ejecutivo del Sistema Nacional de Seguridad Pública (sesnsp), y los propios cuerpos de policía a través de sus páginas web.

En relación con la variable dependiente EF, en la tabla 2 se integran los cuatro indicadores seleccionados, su descripción, escalas de medición, tipo de variable, promedio y fuentes de información.

Tabla 2. Indicadores de la variable independiente eficacia de la policía (EF)

\begin{tabular}{|c|c|c|c|c|}
\hline Indicador & Descripción & Medición / Tipo & $\begin{array}{l}\text { Promedio } \\
\text { por estado }\end{array}$ & Fuente \\
\hline$Y_{1}$ & Robo de automóviles asegurados & $\begin{array}{l}\text { Nominal, por cada } 100000 \\
\text { / Objetiva }\end{array}$ & 632.4 & $\begin{array}{r}(\text { CNSF } \\
2011)\end{array}$ \\
\hline $\mathrm{Y}_{2}$ & Homicidios por habitante & $\begin{array}{l}\text { Nominal, por cada } 100000 \\
\text { / Objetiva }\end{array}$ & 25.3 & $\begin{array}{l}\text { (INEGI } \\
2012 d)\end{array}$ \\
\hline $\mathrm{Y}_{3}$ & $\begin{array}{l}\text { Población que califica como poco o nada efectivo } \\
\text { el desempeño de la policía }\end{array}$ & Porcentual / Subjetiva & $51.8 \%$ & \multirow{2}{*}{$\begin{array}{l}\text { (INEGI } \\
2012 a)\end{array}$} \\
\hline $\mathrm{Y}_{4}$ & Población que percibe sentirse insegura & Porcentual / Subjetiva & $61.8 \%$ & \\
\hline
\end{tabular}

Fuente: Elaboración propia.

El análisis de correlación cruzada entre cada uno de los cuatro indicadores de la variable dependiente EF se muestra en la tabla 3.

Tabla 3. Correlaciones de los indicadores de la variable dependiente EF

\begin{tabular}{lccc}
\hline Indicador & $Y_{1}$ & $Y_{2}$ & $Y_{3}$ \\
\hline$Y_{2}$ & 0.65 & & \\
$Y_{3}$ & 0.31 & 0.15 & \\
$Y_{4}$ & 0.61 & 0.56 & 0.26 \\
\hline
\end{tabular}

Fuente: Elaboración propia.

Se observa que todas las correlaciones tienen signo positivo. Se aprecia una alta correlación significativa entre el número de homicidios y el robo de vehículos 
por estado (65\%), así como entre el número de homicidios y la percepción de inseguridad de los ciudadanos en su estado (61\%). La interpretación es que a mayor robo de automóviles más homicidios, más baja calificación de la policía, y más baja percepción de la seguridad (Gujarati, 1993).

En cuanto a las variables independientes, a pesar de la reciente profesionalización de las policías en México, se reconocieron veintisiete indicadores oficiales relacionados con la organización y el desarrollo de los recursos humanos. Todos cumplen con los primeros cinco criterios de selección de la metodología. En relación al sexto criterio de selección se descartan diecisiete de ellos por la falta de consistencia teórica; cinco porque todos sus coeficientes de correlación tienen signo contrario respecto de los cuatro indicadores de la variable dependiente: $X_{11}$ Policías que opinan que hacen falta horarios más flexibles (SESNSP, 2011a); $X_{19}$ Policías que consideran que en su corporación falta de actitud de servicio por parte de los elementos (SESNSP, 2011a); $X_{23}$ Policías que consideran que en su corporación hay conflictos personales entre elementos (SESNSP, 2011a); $X_{25}$ Policías que tomaron el curso de formación inicial (sesnsp, 2011b); $X_{26}$ Policías que tomaron el curso de capacitación especializada (SESNSP, 2011b). Y doce variables por tener dos o tres coeficientes de correlación con signo contrario respecto de los cuatro indicadores de la variable dependiente: $X_{2}$ Policías preventivos en relación con los policías de investigación (INEGI, 2012e); $X_{3}$ Niveles jerárquicos entre el secretario de Seguridad y el último puesto de policía (manuales de organización de cada estado); $X_{4}$ Nivel jerárquico del puesto cuya responsabilidad principal es la planeación (manuales de organización de cada estado); $X_{6}$ Policías que advierten que el favoritismo es el criterio para un ascenso (SESNSP, 2011b); $\mathrm{X}_{7}$ Policías por cada cien kilómetros cuadrados (INEgI, 2012e); $X_{12}$ Policías que opinan que lo que hace falta son mejores sueldos y prestaciones (SESNSP, $2011 \mathrm{a}$ ); $X_{13}$ Policías que consideran que en su corporación falta organización (sESNSP, 2011a); $X_{16}$ Policías que señalan que lo que más hace falta es un mejor trato y motivación de los superiores (sESNSP, 2011a); $X_{17}$ Policías que indican que lo que más hace falta es más compañerismo (sESNSP, 2011a); $X_{20}$ Policías que consideran que en su corporación hay falta de confianza entre los elementos (SESNSP, 2011a); $X_{21}$ Policías que afirman que en su corporación hay falta de liderazgo de los mandos (sEsnsp, 2011a); $X_{27}$ Policías que opinan que hace falta mayores aportaciones de crecimiento (SESNSP, 2011a).

En la tabla 4 se describen las diez variables que cumplen con los criterios definidos de depuración y selección, su escala de medición, si corresponde a una variable de medición objetiva o subjetiva, y sus fuentes de información. Las diez variables independientes se clasifican en las dos dimensiones de la hipótesis dependiendo de su grado de afinidad: Diseño organizacional (DO) y Desarrollo del factor humano (DH). 
Tabla 4. Variables dependientes que cumplen los criterios de selección definidos

\begin{tabular}{|c|c|c|c|c|}
\hline $\begin{array}{l}\text { Dimensión } \\
\text { /Variable }\end{array}$ & Descripción & Medición / Tipo & $\begin{array}{l}\text { Promedio } \\
\text { por estado }\end{array}$ & Fuente \\
\hline $\mathrm{DO} / \mathrm{X}_{1}$ & $\begin{array}{l}\text { Especialización: Departamentos u organismos con } \\
\text { reporte directo al secretario de Seguridad Pública }\end{array}$ & Nominal / Objetiva & 9.3 & $\begin{array}{l}\text { Páginas } \\
\text { web de } \\
\text { cada policía }\end{array}$ \\
\hline $\mathrm{DO} / \mathrm{X}_{5}$ & $\begin{array}{l}\text { Formalización: Diferencia entre el Registro Nacional } \\
\text { de Personal de Seguridad Pública y el listado nominal }\end{array}$ & $\begin{array}{l}\text { Porcentual / } \\
\text { Objetiva }\end{array}$ & $12.8 \%$ & $\begin{array}{l}\text { (SESNSP, } \\
\text { 2011c) }\end{array}$ \\
\hline $\mathrm{DO} / \mathrm{X}_{8}$ & Remuneraciones: Sueldo mensual de los policías & $\begin{array}{l}\text { Nominal en pesos } \\
\text { / Objetiva }\end{array}$ & $\$ 9,250$ & $\begin{array}{l}\text { (SESNSP, } \\
\text { 2011d) }\end{array}$ \\
\hline $\mathrm{DO} / \mathrm{X}_{9}$ & Tamaño-objetivo: Policías por habitantes del estado & $\begin{array}{l}\text { Nominal, por cada } \\
100000 \text { / Objetiva }\end{array}$ & 141 & $\begin{array}{l}(\text { INEGI, } \\
2012 e)\end{array}$ \\
\hline $\mathrm{DO} / \mathrm{X}_{10}$ & $\begin{array}{l}\text { Tamaño-subjetivo: Policías que consideran que lo } \\
\text { que más hace falta son más elementos }\end{array}$ & $\begin{array}{l}\text { Porcentual / } \\
\text { Subjetiva }\end{array}$ & $14 \%$ & \\
\hline $\mathrm{DO} / \mathrm{X}_{14}$ & $\begin{array}{l}\text { Gobernabilidad: Policías que consideran que en su } \\
\text { corporación existe infiltración del crimen organizado }\end{array}$ & $\begin{array}{l}\text { Porcentual / } \\
\text { Subjetiva }\end{array}$ & $5.1 \%$ & \\
\hline $\mathrm{DH} / \mathrm{X}_{15}$ & $\begin{array}{l}\text { Valores-lealtad: Policías que consideran que lo que } \\
\text { más hace falta es lealtad y sentido de pertenencia }\end{array}$ & $\begin{array}{l}\text { Porcentual/ } \\
\text { Subjetiva }\end{array}$ & $2.8 \%$ & $\begin{array}{c}\text { (SESNSP, } \\
\text { 2011a) }\end{array}$ \\
\hline $\mathrm{DH} / \mathrm{X}_{18}$ & $\begin{array}{l}\text { Valores-integridad: Policías que consideran que en } \\
\text { su corporación existe corrupción de malos elementos }\end{array}$ & $\begin{array}{l}\text { Porcentual / } \\
\text { Subjetiva }\end{array}$ & $14.3 \%$ & \\
\hline $\mathrm{DH} / \mathrm{X}_{22}$ & $\begin{array}{l}\text { Confiabilidad: Policías que consideran que en su } \\
\text { corporación existe negligencia en la actuación entre } \\
\text { los elementos }\end{array}$ & $\begin{array}{l}\text { Porcentual / } \\
\text { Subjetiva }\end{array}$ & $3 \%$ & \\
\hline $\mathrm{DH} / \mathrm{X}_{24}$ & $\begin{array}{l}\text { Capacitación: Policías que consideran que hace } \\
\text { falta más capacitación }\end{array}$ & $\begin{array}{l}\text { Porcentual / } \\
\text { Subjetiva }\end{array}$ & $29.4 \%$ & $\begin{array}{l}\text { (SESNSP, } \\
\text { 2011b) }\end{array}$ \\
\hline
\end{tabular}

DO: Diseño organizacional; DH: Desarrollo del factor humano.

Fuente: Elaboración propia.

Sobre la validez del modelo, destaca la escasez de datos y la falta de consistencia técnica de algunos de los localizados, por lo que nuestra evaluación de la eficacia de las policías se limitó a las dimensiones de organización y de recursos humanos. Por ejemplo, se excluyeron importantes dimensiones como a) equipamiento y equipo, $b$ ) presupuesto, $c$ ) respeto a los derechos humanos, y d) sistemas de información e inteligencia; todas las cuales se detallan en la tabla 1. Por tal hecho, esta investigación no pretende construir un modelo de validez general, sino solo identificar la posible significancia de las dos dimensiones señaladas en relación con la eficacia de la policía.

Acerca de la confiabilidad de las dimensiones se buscó hacer algún tipo de factorización de las variables independientes, pero eso no es recomendable debido a que los valores de alfa de Cronbach encontrados fueron poco significativos (DO con A.C. = -0.01351; DH con A.C. = 0.1499). Más bien lo que debe hacerse, aunado a que los datos vienen de fuentes oficiales, es un análisis independiente de las variables, sin consolidarlas, lo cual es viable siempre y cuando haya estimaciones de fiabilidad (Hair, Anderson, Tatham \& Black, 1999).

De la revisión de los 448 datos de los indicadores de las diez variables independientes y de los cuatro indicadores de la variable dependiente, se corrigieron siete datos atípicos y se determinaron veintiún faltantes. En total 
representaron el 6.25\% de la base de datos del análisis cualitativo, lo cual se consideró como un margen tolerable para el estadístico (Arias, 2008; Frane, 1976; Johnston, 2000; McKnight, McKnight, Sidani \& Figueredo, 2007; Osborne \& Overbay, 2004).

$\mathrm{Al}$ aplicar el análisis de regresión multivariada, se observó que la variable dependiente EF está significativamente relacionada con el DO ( $\mathrm{p}$-value $<0.05$; $\left.\mathrm{R}=0.756 ; \mathrm{R}^{2} \mathrm{aj}=0.469\right)$. En particular las variables $X_{1}$ Especialización, $X_{8} R e-$ muneraciones, $X_{10}$ Tamaño-Subjetivo, y $X_{14}$ Gobernabilidad, ya que cada una de estas reportan una aportación significativa ( $\mathrm{p}$-value $<0.10$ ), como se muestra en la tabla 5.

Tabla 5. Análisis de regresión entre la eficacia de las policías y las variables del diseño organizacional

\begin{tabular}{|c|c|c|c|c|c|c|c|c|c|}
\hline \multirow{2}{*}{$R$} & \multirow{2}{*}{$R^{2}$} & \multirow{2}{*}{$\begin{array}{c}R^{2} \\
\text { ajustado }\end{array}$} & \multirow{2}{*}{\multicolumn{2}{|c|}{ Error estándar de la estimación }} & \multicolumn{5}{|c|}{ Estadísticos de cambio } \\
\hline & & & & & $n$ & $F$ & $g / 1$ & $g / 2$ & Sig \\
\hline .756 & .572 & .469 & .31797 & & 32 & 5.565 & 6 & 25 & .001 \\
\hline \multirow{2}{*}{ Modelo } & & \multicolumn{2}{|c|}{$\begin{array}{l}\text { Coeficientes no } \\
\text { estandarizados }\end{array}$} & \multicolumn{2}{|c|}{$\begin{array}{c}\text { Coeficientes } \\
\text { estandarizados }\end{array}$} & \multirow{2}{*}{$t$} & \multirow{2}{*}{ Sig. } & & \multirow{2}{*}{ VIF } \\
\hline & & $B$ & $\begin{array}{c}\text { Error } \\
\text { estándar }\end{array}$ & \multicolumn{2}{|c|}{ Beta } & & & & \\
\hline \multicolumn{2}{|c|}{ (Constante) } & .154 & .353 & & & .436 & \multicolumn{2}{|l|}{.667} & \\
\hline \multicolumn{2}{|c|}{$X_{1}$ Especialización } & .055 & .019 & \multicolumn{2}{|c|}{.424} & 2.951 & .007 & & 1.206 \\
\hline \multicolumn{2}{|c|}{$X_{5}$ Formalización } & .002 & .006 & \multicolumn{2}{|c|}{.062} & .405 & .689 & & 1.345 \\
\hline \multicolumn{2}{|c|}{$\mathrm{X}_{8}$ Remuneraciones } & $-4.092 E-5$ & .000 & \multicolumn{2}{|c|}{-.293} & -1.715 & .099 & & 1.704 \\
\hline \multicolumn{2}{|c|}{$X_{9}$ Tamaño-Objetivo } & .000 & .001 & \multicolumn{2}{|c|}{-.095} & -.642 & .527 & & 1.287 \\
\hline \multicolumn{2}{|c|}{$X_{10}$ Tamaño-Subjetivo } & .034 & .011 & \multicolumn{2}{|c|}{.518} & 3.226 & .003 & & 1.505 \\
\hline \multicolumn{2}{|c|}{$X_{14}$ Gobernabilidad } & .046 & .018 & \multicolumn{2}{|c|}{.353} & 2.508 & .019 & & 1.153 \\
\hline
\end{tabular}

Adicionalmente, se observa en la tabla 5 que las variables del diseño organizacional con mayor influencia en el modelo sobre la eficacia de las policías son $X_{10}$ Tamaño-Subjetivo (Beta $\left.=0.518\right)$, y $X_{1}$ Especialización, la cual es una variable objetiva $($ Beta $=0.424)$. En el modelo no se observan en las variables problemas de multicolinealidad (VIF $<5$ ). Destaca el signo negativo que se obtuvo de la variable $X_{8}$ Remuneraciones.

La regresión multivariada de la variable dependiente EF está significativamente relacionada con el desarrollo del factor humano ( $\mathrm{p}$-value $<0.05 ; \mathrm{R}=$ $\left.0.626 ; \mathrm{R}^{2} \mathrm{aj}=0.302\right)$. En particular con las variables $X_{15}$ Valores-Lealtad, y $X_{22}$ Confiabilidad; estas presentan una aportación significativa ( $\mathrm{p}$-value $<0.10$ ), como se muestra en la tabla 6. 
Tabla 6. Análisis de regresión entre la eficacia de las policías y las variables del desarrollo del factor humano

\begin{tabular}{|c|c|c|c|c|c|c|c|c|c|}
\hline \multirow{2}{*}{$\mathrm{R}$} & \multirow{2}{*}{$\mathrm{R}^{2}$} & \multirow{2}{*}{$\begin{array}{c}\mathrm{R}^{2} \\
\text { ajustado }\end{array}$} & \multirow{2}{*}{\multicolumn{2}{|c|}{ Error estándar de la estimación }} & \multicolumn{5}{|c|}{ Estadísticos de cambio } \\
\hline & & & & & $n$ & $\mathrm{~F}$ & $g l 1$ & $\mathrm{gl} 2$ & Sig \\
\hline .626 & .392 & .302 & & 6446 & 32 & 4.361 & 4 & 27 & .008 \\
\hline \multirow{2}{*}{ Modelo } & & & \multicolumn{2}{|c|}{$\begin{array}{l}\text { Coeficientes no } \\
\text { estandarizados }\end{array}$} & $\begin{array}{l}\text { Coeficientes } \\
\text { standarizados }\end{array}$ & \multirow{2}{*}{\multicolumn{2}{|c|}{$t$}} & \multirow{2}{*}{ Sig. } & \multirow{2}{*}{ VIF } \\
\hline & & & $B$ & $\begin{array}{c}\text { Error } \\
\text { estándar }\end{array}$ & Beta & & & & \\
\hline \multicolumn{2}{|c|}{ (Constante) } & & -.377 & .363 & & \multicolumn{2}{|l|}{-1.037} & .309 & \\
\hline \multicolumn{2}{|c|}{$X_{15}$ Valores-Lealtad } & & .154 & .060 & .403 & \multicolumn{2}{|l|}{2.567} & .016 & 1.094 \\
\hline \multicolumn{3}{|c|}{$X_{16}$ Valores-Integridad } & .014 & .012 & .193 & \multicolumn{2}{|l|}{1.195} & .242 & 1.162 \\
\hline \multicolumn{3}{|c|}{$X_{22}$ Confiabilidad } & .150 & .064 & .383 & \multicolumn{2}{|l|}{2.360} & .026 & 1.170 \\
\hline \multicolumn{3}{|c|}{$X_{24}$ Capacitación } & .011 & .008 & .196 & \multicolumn{2}{|l|}{1.300} & .205 & 1.014 \\
\hline
\end{tabular}

Fuente: Elaboración propia.

Se observa también en la tabla 6 que las variables del desarrollo del factor humano con mayor influencia en el modelo sobre la eficacia de las policías son $X_{15}$ Valores-Lealtad $($ Beta $=0.403)$, y $X_{22}$ Confiabilidad $($ Beta $=0.383)$; ambas son variables subjetivas. En el modelo, las variables no muestran problemas de multicolinealidad (VIF < 5).

Si se corre la regresión de la variable dependiente EF con el total de las variables independientes, el producto es que el modelo en su conjunto está significativamente relacionado ( $p$-value $<0.05 ; R=0.822 ; R^{2} a j=0.543$ ). Se advierte además que se mantiene la significancia individual de las variables $X_{1}$ Especialización $($ Beta $=0.399)$, y $X_{10}$ Tamaño-Subjetivo $($ Beta $=$ 0.442 ).

Por otra parte, al aplicar la ecuación de regresión obtenida para la eficacia de la policía y usando solo las variables independientes significativas, se concluye que las entidades federativas con cuerpos de policía preventivos de alta eficacia son Yucatán, Aguascalientes, Baja California Sur, Campeche y Querétaro. Estas entidades reportan menos de siete homicidios por cada cien mil habitantes al año, menos de 274 robos por cada cien mil automóviles asegurados al año, menos del $51 \%$ de la población califica a la policía estatal como poco o nada efectiva en su desempeño y menos del $47 \%$ de la población percibe sentirse insegura en su estado.

En Yucatán, solo el 1\% de los policías considera que lo que más hace falta es lealtad y sentido de pertenencia. En Querétaro, solo el 2\% de los policías opinan que en su corporación existe negligencia en la actuación de los elementos. Y en Baja California Sur, solo 6\% de los policías señalan que lo que más hace falta son más elementos. 
Dos hallazgos no planeados de esta investigación surgieron al correr un par de regresiones adicionales con el método best subsets regression, usando como variable dependiente cada uno de los indicadores $\left(\mathrm{Y}_{\mathrm{i}}\right)$ de la variable dependiente eficacia de la policía EF, y como independientes todas las variables seleccionadas del DO y DH. Dichas ecuaciones son:

a) La variable independiente $Y_{2}$ Homicidios versus $X_{1}$ Especialización, $X_{8}$ Remuneraciones, $X_{10}$ Tamaño-Subjetivo, $X_{14}$ Gobernabilidad, $X_{15}$ Valores-Lealtad, $X_{22}$ Confiabilidad. El análisis se resume en la tabla 7.

Tabla 7. Análisis de regresión del número de homicidios por estado $\left(\mathrm{Y}_{2}\right)$

\begin{tabular}{|c|c|c|c|c|c|c|c|c|}
\hline \multirow{2}{*}{$\mathrm{R}$} & \multirow{2}{*}{$\mathrm{R}^{2}$} & \multirow{2}{*}{$\begin{array}{c}\mathrm{R}^{2} \\
\text { ajustado }\end{array}$} & \multirow{2}{*}{ Error estándar de la estimación } & \multicolumn{5}{|c|}{ Estadísticos de cambio } \\
\hline & & & & $\mathrm{n}$ & $\mathrm{F}$ & $\mathrm{gl}$ & $\mathrm{gl} 2$ & Sig. \\
\hline .858 & .739 & .672 & .52706 & 32 & 11.597 & 6 & 25 & .000 \\
\hline \multirow{2}{*}{ Modelo } & & \multicolumn{2}{|c|}{$\begin{array}{l}\text { Coeficientes no } \\
\text { estandarizados }\end{array}$} & $\begin{array}{l}\text { Coeficientes } \\
\text { standarizados }\end{array}$ & \multirow{2}{*}{$t$} & \multirow{2}{*}{\multicolumn{2}{|c|}{ Sig. }} & \multirow{2}{*}{ VIF } \\
\hline & & $B$ & $\begin{array}{c}\text { Error } \\
\text { estándar }\end{array}$ & Beta & & & & \\
\hline \multicolumn{2}{|c|}{ (Constante) } & -1.858 & .559 & & -3.326 & \multicolumn{2}{|r|}{.003} & 1.254 \\
\hline \multicolumn{2}{|c|}{$x_{1}$ Especialización } & .130 & .031 & .476 & 4.131 & \multicolumn{2}{|r|}{.000} & 1.755 \\
\hline \multicolumn{2}{|c|}{$\mathrm{X}_{8}$ Remuneraciones } & $-8.992 E-5$ & .000 & -.305 & -2.241 & \multicolumn{2}{|r|}{.034} & 1.500 \\
\hline \multicolumn{2}{|c|}{$X_{10}$ Tamaño-Subjetivo } & .065 & .017 & .470 & 3.731 & \multicolumn{2}{|r|}{.001} & 1.279 \\
\hline \multicolumn{2}{|c|}{$X_{14}$ Gobernabilidad } & .085 & .032 & .312 & 2.682 & \multicolumn{2}{|r|}{.013} & 1.607 \\
\hline \multicolumn{2}{|c|}{$X_{15}$ Valores-Lealtad } & .221 & .105 & .274 & 2.105 & \multicolumn{2}{|r|}{.046} & 1.184 \\
\hline \multicolumn{2}{|c|}{$X_{22}$ Confiabilidad } & .167 & .093 & .202 & 1.803 & \multicolumn{2}{|r|}{.083} & 1.254 \\
\hline
\end{tabular}

La regresión de la variable dependiente $Y_{2}$ Homicidios está significativamente relacionada con algunas de las variables del $\mathrm{DO}$ y del desarrollo del factor humano ( $\mathrm{p}$-value $\left.<0.000 ; \mathrm{R}=0.858 ; \mathrm{R}^{2} \mathrm{aj}=0.739\right)$, y todas las variables tienen una aportación significativa ( $\mathrm{p}$-value $<0.10$ ). Las que tienen una mayor influencia en el modelo sobre los homicidios son $X_{1}$ Especialización, una variable objetiva $($ Beta $=0.476), y X_{10}$ Tamaño subjetivo $($ Beta $=0.70)$. En este modelo no hay problemas de multicolinealidad (VIF < 5) en las variables. Un dato destacado es el signo negativo que se obtuvo en la variable $X_{8}$ Remuneraciones (con una P-value > 0.005).

b) La variable independiente Homicidios versus $X_{1}$ Especialización, $X_{8}$ Remuneraciones, $X_{10}$ Tamaño-Subjetivo, $X_{14}$ Gobernabilidad, $X_{15}$ Valores-Lealtad, $X_{22}$ Confiabilidad. El análisis se encuentra en la tabla 8. 
Tabla 8. Análisis de regresión de la percepción de la inseguridad por estado $\left(\mathrm{Y}_{4}\right)$

\begin{tabular}{|c|c|c|c|c|c|c|c|c|c|}
\hline \multirow{2}{*}{$\mathrm{R}$} & \multirow{2}{*}{$\mathrm{R}^{2}$} & \multirow{2}{*}{$\begin{array}{c}R^{2} \\
\text { ajustado }\end{array}$} & \multirow{2}{*}{\multicolumn{2}{|c|}{ Error estándar de la estimación }} & \multicolumn{5}{|c|}{ Estadísticos de cambio } \\
\hline & & & & & $n$ & $\mathrm{~F}$ & gl1 & gl2 & Sig. \\
\hline .729 & .532 & .442 & & 636 & 32 & 5.910 & 5 & 26 & .001 \\
\hline \multirow{2}{*}{ Modelo } & & & \multicolumn{2}{|c|}{$\begin{array}{l}\text { Coeficientes no } \\
\text { estandarizados }\end{array}$} & $\begin{array}{l}\text { Coeficientes } \\
\text { estandarizados }\end{array}$ & \multirow{2}{*}{\multicolumn{2}{|c|}{$t$}} & \multirow{2}{*}{ Sig. } & \multirow{2}{*}{ VIF } \\
\hline & & & $B$ & $\begin{array}{c}\text { Error } \\
\text { estándar }\end{array}$ & Beta & & & & \\
\hline \multicolumn{2}{|c|}{ (Constante) } & & -.149 & .288 & & \multicolumn{2}{|l|}{-.519} & .608 & \\
\hline \multicolumn{2}{|c|}{$X_{1}$ Especialización } & & .036 & .013 & .402 & \multicolumn{2}{|l|}{2.821} & .009 & 1.130 \\
\hline \multicolumn{2}{|c|}{$\mathrm{X}_{8}$ Remuneraciones } & & $-1.996 \mathrm{E}-5$ & .000 & -.206 & \multicolumn{2}{|l|}{-1.306} & .203 & 1.381 \\
\hline \multicolumn{2}{|c|}{$X_{15}$ Valores-Lealtad } & & .084 & .042 & .318 & \multicolumn{2}{|l|}{2.019} & .054 & 1.382 \\
\hline \multicolumn{2}{|c|}{$X_{22}$ Confiabilidad } & & .109 & .039 & .399 & \multicolumn{2}{|l|}{2.820} & .009 & 1.110 \\
\hline \multicolumn{2}{|c|}{ X $_{24}$ Capacitación } & & .015 & .005 & .406 & \multicolumn{2}{|l|}{2.980} & .006 & 1.033 \\
\hline
\end{tabular}

Fuente: Elaboración propia.

La regresión de la variable dependiente $Y_{4}$ Percepción de la inseguridad está significativamente relacionada con varias de las variables del DO y del desarrollo del factor humano ( $p$-value $<0.000 ; R=0.729 ; R^{2} a j=0.442$ ), en donde cuatro de cinco variables presentan una aportación significativa ( $\mathrm{p}$-value $<$ 0.10). Las que tienen una mayor influencia en el modelo sobre la percepción de la inseguridad ciudadana son $X_{24}$ Capacitación, una variable subjetiva (Beta $=0.406)$, y $X_{1}$ Especialización, una variable objetiva (Beta $\left.=0.402\right)$. No se observan problemas de multicolinealidad $(\mathrm{VIF}<5)$ en las variables. Y un dato que llama la atención es el signo negativo que se obtuvo en la variable $X_{8}$ Remuneraciones, sin que sea significativa ( $\mathrm{P}$-value $>0.10$ ).

Todo esto indica para México que la policía entre más especializada esté, más grande sea, y más desarrollado tenga el sentido de lealtad y el sentimiento de confiabilidad interna, entonces podría haber menos homicidios. Este hallazgo parece reforzar la idea de que debe existir un mando único en los estados de la república mexicana, lo cual a pesar de ser una iniciativa presidencial no ha podido consolidarse.

Para las combinaciones de regresión con $Y_{1}$ Robo de automóviles y $Y_{3}$ Evaluación ciudadana del desempeño, analizadas en lo individual, no se encontraron modelos interesantes.

Un hallazgo perturbador fue constatar la correlación de $0.729 \%$ entre los indicadores de las variables causales Lealtad, Confiabilidad y Capacitación $\left(\mathrm{X}_{15}, \mathrm{X}_{22}\right)$ con el indicador Población que percibe sentirse insegura en su estado $\left(\mathrm{Y}_{4}\right)$. Esto significa que cuando hay una buena capacitación para dar un sentido de lealtad y confiabilidad en el cuerpo de policía debería haber una buena percepción ciudadana, pero hoy existe una sensación inversa en la población. 
Esta se debería sentir más segura pero no es así; las encuestas de opinión señalan lo contrario.

Las relaciones significativas determinadas por el análisis MAR se presentan en el gráfico 1 .

Gráfico 1. Relaciones significativas entre las variables de diseño organizacional y las de desarrollo del factor humano y la variable eficacia de la policía

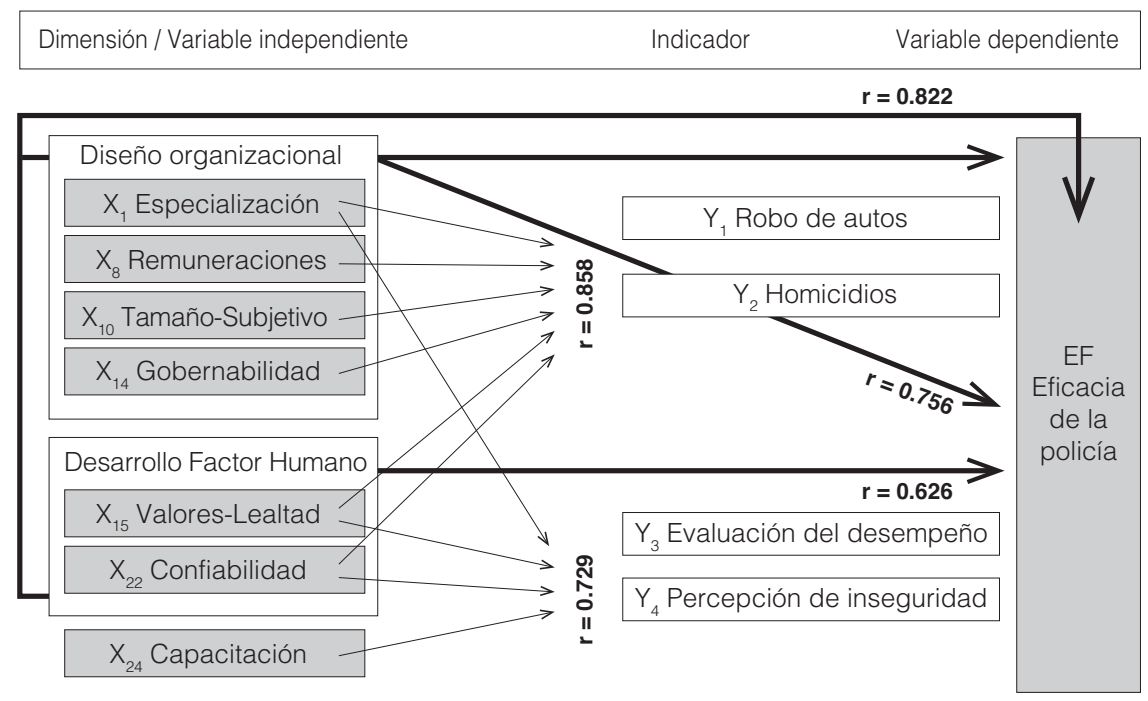

Nota: Solo se presentan las flechas de las variables con significancia $\mathrm{P}<0.10$.

Fuente: Elaboración propia.

\section{Discusión}

La complejidad para poder evaluar a la policía en México se origina en la escasa denuncia ciudadana de los delitos. Como se citó arriba, la cifra negra para 2011 en México fue del 91.6\%. Sumado a esto, otro problema es la falta de disponibilidad de los indicadores policíacos para elaborar procesos de investigación. Ello motivó que algunos de los datos de este estudio se obtuvieran vía requerimientos de transparencia. Esta limitante de indicadores objetivos incide en la correcta evaluación de la gestión de las policías, y deja a los investigadores para su uso pocos indicadores objetivos confiables, como lo son los homicidios y el robo de autos asegurados, mismos que se han utilizado aquí como parte integrante de la variable dependiente. 
Ahora bien, en relación la literatura revisada, nuestros resultados coinciden y discrepan en varios aspectos. A saber:

1. Se confirman las observaciones de los estudios de Wells \& Falcone (2005) y de Wilson (2005) relativas a los diferentes tamaños y ubicaciones de las organizaciones de policía, ya que a partir de los hallazgos de este artículo se identificó que la percepción del volumen de agentes disponibles $\left(\mathrm{X}_{10}\right)$ es un factor crítico para la eficacia de la policía, a diferencia de la dispersión geográfica de policías por kilómetro cuadrado $\left(\mathrm{X}_{7}\right)$, la cual tiene nula aportación; esto sugiere que se deben configurar grandes corporaciones en las ciudades y pequeños destacamentos en las poblaciones.

2. Se confirman los estudios de Wilson (2005) en relación a lo establecido por Gujarati (1993) en cuanto a la débil relación estadística que existe entre las remuneraciones y la eficacia en el desempeño de los policías $\left(\mathrm{X}_{8}\right.$ $\mathrm{y} \mathrm{X}_{12}$ ).

3. Acerca del estudio de Rico \& Chinchilla (2002), en el que proponen que el índice de gestión de eficacia de una organización de policía requiere incluir el grado de competencia en las actividades de los agentes, se ratifica dado que se encontró que el porcentaje de policías que consideran que en su corporación existe negligencia en la actuación entre los elementos $\left(\mathrm{X}_{22}\right)$ es una variable significativa.

4. Sorpresivamente se obtuvo una divergencia vinculada a las aportaciones de la capacitación de los policías de Mastrofski (2007), Newburn (2007), Oliveira-Muniz \& Proença (2007), Purpura (2001), Salamanca (2004) y Tyler (2011), ya que en esta investigación, tanto la variable $\left(\mathrm{X}_{25}\right)$ Policias que tomaron el curso de formación inicial, como la variable $\left(\mathrm{X}_{26}\right)$ Policias que tomaron el curso de capacitación especializada, fueron descartadas, debido a que ambas tuvieron correlaciones negativas respecto a todos los indicadores de seguridad. Esta divergencia sugiere que los cursos que se está impartiendo en México no cuenten con la calidad y efectividad requerida, sobre todo en cuanto al uso de la fuerza y al desarrollo del criterio discrecional de actuación de los policías en la calle, las cuales son dos características de los cursos reflejadas por los investigadores revisados.

Esto puede que se deba a la pésima imagen pública de la policía en todos los estados de la república mexicana ya que, frecuentemente, en crímenes de alto impacto suelen estos estar asociados a expolicías y policías en activo. El caso más simbólico es el de los 43 estudiantes de la Normal Rural de Ayotzinapa, Guerrero, en el cual de los 111 detenidos acusados hay sesenta policías. 
5. Igualmente se obtuvieron resultados contradictorios en relación con las aportaciones de Mastrofski (2007) y de Tyler \& Huo (2002) acerca de la necesidad de acompañar a la policía con un programa sistemático de evaluación, ya que tanto la variable $\left(\mathrm{X}_{6}\right)$ Policías que consideran que el favoritismo es el criterio para un ascenso, como la variable $\left(\mathrm{X}_{27}\right)$ Policias que opina que lo que hace falta son mayores aportaciones de crecimiento, fueron descartadas por su nula consistencia teórica. Esta contradicción sugiere que tanto los procesos de evaluación como de promoción que se están dando en la policía en México requieren revisarse en profundidad.

La investigación arroja dos aportaciones que se desprenden de los resultados del análisis cuantitativo para que las policías estatales incrementen significativamente su eficacia:

a) En lo organizacional, especializar su estructura de tal manera que se cuente con el número de puestos correctos con reporte directo al secretario de Seguridad Pública, además de tomar acciones para reducir el porcentaje de policías que suponen que lo que más hace falta son más elementos, y el que considera que en su corporación hay infiltración del crimen organizado.

b) En materia de recursos humanos, tomar acciones para reducir el porcentaje de policías que opinan que lo que más hace falta es lealtad y sentido de pertenencia, y el que supone que en su corporación existe negligencia en la actuación de los elementos.

Una aportación relevante es comprobar que, con base en los resultados del estudio, para prevenir, y en su caso reducir, el número de homicidios por habitante se requiere que las policías estatales vigilen cuatro puntos: $a$ ) la especialización de funciones de los departamentos u organismos con reporte directo al secretario de Seguridad Pública Estatal; $b$ ) la falta de agentes; $c$ ) la infiltración del crimen organizado, y d) la lealtad y sentido de pertenencia, lo cual sería interesante abordar a partir de lo realizado bajo el modelo de La Policía de Aseguramiento de Barrio, el cual se ha estudiado con profundidad en Reino Unido (Bertaccini, 2011).

Esta investigación propone la creación de un índice mixto cuantitativo y cualitativo que podría integrarse del siguiente modo: a) cuantitativo: el número de homicidios, el número de autos asegurados robados, y $b$ ) cualitativo: la percepción ciudadana de inseguridad en su colonia, y la calificación ciudadana de la actuación de la policía. Este índice compuesto sería útil para realizar comparaciones entre varios países o identificar otras variables significativas en eficacia. 
Una limitación del presente estudio, tarea que puede continuarse en futuras investigaciones, es que se han excluido otras dimensiones identificadas en la literatura tales como equipamiento y equipo, presupuesto, respeto a los derechos humanos y sistemas de información e inteligencia, las cuales ayudarían a enriquecer el modelo. Otro tema pendiente que se vincula a la relación entre capacitación y eficacia de los cuerpos de policía, fue que los resultados de esta investigación no han sido contundentes, pues con la información oficial disponible no se lograron conclusiones significativas en el tema. Así mismo quedaron por identificar las variables que impactarían la calificación que la ciudadana da a sus policías preventivos, ya que con la información disponible no se encontró variable alguna que tuviera un impacto significativo considerable.

\section{Referencias}

Abid, A., Sarwar, A., Imran, K., Jabbar, A. \& Hannan, A. (2013). Effect of Job Design on Employee Satisfaction (A Study of Fertilizer Companies Listed in Lahore Stock Exchange). European Journal of Business and Management, 5(19), 1-7.

Alpert, G. (1994). Study Group on Criminal Justice Performance Measures: Performance Measures for the Criminal Justice System. Princeton, Estados Unidos: Department of Justice, Bureau of Justice Statistics.

Amadi, E. N. (2014). A Qualitative Analysis of Community Policing in the United States. American International Journal of Contemporary Research, 4(1), 19-26.

Arias, B. (2008). Desarrollo de un ejemplo de análisis factorial confirmatorio con LISREL, AMOS y SAS. Espańa: Universidad de Valladolid. Seminario de Actualización en Investigación sobre Discapacidad SAID. Recuperado el 19 de abril de 2017, de http://riberdis.cedd .net/bitstream/handle/11181/3270/Metodologia_en_investigacion_sobre_discapacidad .pdf?sequence $=2 \#$ page $=76$

Belamaric, R., Arrastía, F. \& Cañizares, R. (2001). Perfeccionamiento empresarial: realidades y retos. La Habana: Editorial de Ciencias Sociales.

Benítez, R. G. (2010). Seguridad y defensa en América del Norte: Nuevos dilemas geopolíticos. El Salvador: Fundación Guillermo Manuel Ungo/Latin American Program, Woodrow Wilson Internacional Center for Scholars.

Bertaccini, D. (2011). La politica di polizia. Bolonia: Bononia University Press. 
Cano, I. (2003). La policía y su evaluación. Propuestas para la construcción de indicadores de evaluación en el trabajo policial. Centro de Estudios para el Desarrollo, Área Seguridad Ciudadana, Santiago de Chile. Recuperado el 19 de abril de 2017, de http://bibliotk.iep.org.pe/ bib_img/21075-8-1.pdf

Castillo, R. (2012). Desarrollo del capital humano en las organizaciones. México: Red Tercer Milenio.

Chiavenato, I. (2000). Administración de recursos humanos: el capital humano de las organizaciones. Bogotá: McGraw-Hill.

CNSF. (2011). Información estadística de Autos. Recuperado el 30 de junio de 2013, de http:// www.cnsf.gob.mx/InformacionEstadistica/DetalladaSeguros/Paginas/Automoviles.aspx

Cockcroft, T. (2013). Police culture. Themes and concepts. Nueva York: Routledge.

Cook, C. \& Hunsaker, P. (2001). Management and organizational behavior. Estados Unidos: McGraw-Hill/Irwin.

Crank, J. \& Crank, J. P. (2014). Understanding police culture. Nueva York: Routledge.

Cruz, P., Maganda, M., Hernández, J. \& González, V. (2015). Elementos para la evaluación y gestión de la complejidad situacional sobre la inseguridad y la violencia en México. Trabajo Social UNAM, (7), 35-47.

Daft, R. (2015). Teoría y diseño organizacional. México: Cengage.

Davenport, T. O. (2000). Capital humano: Creando ventajas competitivas a través de las personas. Barcelona: Ediciones Gestión 2000.

Decker, S. H. \& Pyrooz, D. C. (2010). On the Validity and Reliability of Gang Homicide: A Comparison of Disparate Sources. Homicide Studies, 14(4), 359-376.

Endrikat, J., Guenther, E. \& Hoppe, H. (2014). Making sense of conflicting empirical findings: A meta-analytic review of the relationship between corporate environmental and financial performance. European Management Journal, 32(5), 735-751.

Frane, J. W. (1976). Some simple procedures for handling missing data in multivariate analysis. Psychometrika, 41(3), 409-415.

Fried, Y. \& Ferris, G. R. (1987). The validity of the job characteristics model: A review and meta-analysis. Personnel psychology, 40(2), 287-322. 
García-Luna, G. (2006). ¿Por qué 1,661 corporaciones de policía no bastan? México: Kan sasana Printer.

Gibson, J. L., Ivancevich, J. M., Konopaske, R. \& Donnelly, J. H. (2011). Organizaciones: comportamiento, estructura, procesos. México: McGraw-Hill.

Gooding, R. Z. \& Wagner III, J. A. (1985). A meta-analytic review of the relationship between size and performance: The productivity and efficiency of organizations and their subunits. Administrative Science Quarterly, 30(4), 462-481.

Griffin, R., Phillips, J. M. \& Gully, S. M. (2017). Organizational behavior. México: Nelson Education.

Gujarati, D. N. (1993). Econometría. México: McGraw Hill Interamericana.

Hair, J. F., Anderson, R. E., Tatham, R. L. \& Black, W. C. (1999). Análisis multivariable. Madrid: Prentice Hall.

Heath, L. (1984). Impact of newspaper crime reports on fear of crime: Multimethodological investigation. Journal of Personality and social Psychology, 47(2), 263.

Hellriegel, D., Jackson, S. \& Slocum, J. (2007). Managing: A competency-based approach. USA: Thomson Learning.

Hernández-Sampieri, R., Fernández, C. \& Baptista, P. (2006). Metodología de la investigación. México: McGraw Hill Interamericana.

ICESI. (2010a). Victimización, incidencia y cifra negra en México: Análisis de la ENSI-6/2009. Porcentaje de delitos denunciados ante el Ministerio Público. México: Instituto Ciudadano de Estudios sobre la Inseguridad. Recuperado el 19 de abril de 2017, de http://www.casede .org/index.php/bibliotecacasede/encuestas-estadisticas-y-datos-duros/155-cuadernos-del -icesi-8-victimizacion-incidencia-y-cifra-negra-en-mexico

ICESI. (2016b). Mortalidad por homicidios en México. México: Instituto Ciudadano para el Estudio de la Inseguridad. Recuperado el 19 de abril de 2017, de http://seguridadenperspectiva .blogspot.com/2010/02/cuadernos-del-icesi-6-mortalidad-por.html

INEgr. (2012a). Encuesta Nacional de Victimización y Percepción sobre Seguridad Pública. Recuperado el 30 del junio de 2013, de http://www.inegi.org.mx/est/contenidos/proyectos/ encuestas/hogares/regulares/envipe/default.aspx 
Inegi. (2012b). Encuesta Continua sobre la Percepción de la Seguridad Pública. Recuperado el 30 de junio de 2013, de http://www.inegi.org.mx/est/contenidos/proyectos/encuestas /hogares/regulares/ecosep/default.aspx

INEGI. (2012c). Boletín de prensa número 339/12. Aguascalientes: INEGI. Recuperado el 30 de junio de 2013, de http://www.inegi.org.mx/est/contenidos/proyectos/encuestas/hogares /regulares/envipe/envipe2012/default.aspx?_file=/inegi/contenidos/espanol/prensa/ boletines/boletin/comunicados/especiales/2012/septiembre/comunica5.pdf

INEGI. (2012d, 20 de agosto). En 2011 se registraron 27 mil 199 homicidios. Aguascalientes: INEGI. Boletín de prensa núm. 310/12. Recuperado el 30 de junio de 2013, de http:// www.inegi.org.mx/inegi/contenidos/espanol/prensa/boletines/Boletin/Comunicados/Especiales/2012/Agosto/comunica29.pdf

Inegi. (2012e). Censo Nacional de Gobierno, Seguridad Pública y Sistema Penitenciario Estatales 2012. Recuperado el 30 de junio de 2013, de http://www.beta.inegi.org.mx/proyectos/censosgobierno/estatal/cngspspe/2017/

Johnston, D. E. (2000). Métodos multivariados aplicados al análisis de datos. México: Thomson.

Johnston, J. J. (1972). Econometric methods. Nueva York: McGraw-Hill.

Kennedy, P. (2002). Sinning in the basement: What are the rules? The ten commandments of applied econometrics. Journal of Economic Surveys, 16(4), 569-89.

Kennedy, P. E. (2005). Oh no! I got the wrong sign! What should I do? The Journal of Economic Education, (36), (77-92).

Lawrence, P. R. \& Lorsch, J. W. (1986). Organization and environment: Managing integration and differentiation. Estados Unidos: Harvard Business School Press.

Liu, Y., Guo, J. \& Chi, N. (2015). The antecedents and performance consequences of proactive environmental strategy: A meta-analytic review of national contingency. Management and Organization Review, 11(3), 521-557.

Loubet Del Bayle, J.-L. (2012). De la police et du contrôle social. París: Les editions du CuRF.

Maella, P. (2010). Las variables y las conductas de la eficacia personal. (Ocasional Paper, núm. 174). Barcelona: IESE Business School, Universidad de Navarra.

Mastrofski, S. D. (2007). Police Organization and Management Issues for the Next Decade. Washington: National Institute of Justice (NIJ). Recuperado el 19 de abril de 2017, de https:// www.ncjrs.gov/pdffiles1/nij/grants/218584.pdf 
Mastrofski, S. D. (2004). Controlling Street-level police discretion. Annals of the American Academy of Political and Social Science, (593), 100-118.

Mastrofski, S. (1999). Policing For People. Ideas in American Policing. Washington: Police Foundation. Recuperado el 19 de abril de 2017, de https:/www.policefoundation.org/wp -content/uploads/2015/06/Mastrofski-1999-Policing-For-People.pdf

McKnight, P. E., McKnight, K. M., Sidani, S. \& Figueredo, A. J. (2007). Missing Data: A Gentle Introduction. Nueva York: The Guilford Press.

Medina Ariza, J. (2011). Políticas y estrategias de prevención del delito y Seguridad ciudadana. Madrid: Edisofer.

Mintzberg, H. (1979). The structuring of organizations (Vol. 203). NJ: Prentice Hall.

Muñiz, J. (2000). Teoría clásica de los tests. Madrid: Pirámide.

Muniz, J. \& Proença, D. (2007). Bases Conceituais de Métricas e Padrões de Medida de Desempenho Policial. En Carusso, H., Muniz, J. \& Carballo Blanco, A. C. (Coords.). Polícia, estado e sociedade: práticas e saberes latino-americanos: Policia e policía (págs. 231-280). Río de Janeiro: Publit Soluçôes Editoriais. Recuperado el 19 de abril de 2017, de http://pm.al .gov.br/intra/downloads/bc_policial/pol_02.pdf

National Research Council. (2004). Fairness and Effectiveness in Policing: The Evidence. (Skogan, W. G. \& Frydl, K., Eds.). Washington: The National Academies Press.

Nelson, D. \& Quick, J. (2013). CORG: Comportamiento Organizacional. México: Cengage Learning.

Newburn, T. (2007). Criminology. Cullompton, Portland: Willan Publishing.

O’Neill, M., Marks, M. \& Singh, A. (2007). Police Occupational Culture: New Debates and Directions. Nueva York: Elsevier.

oECD. (2017). Better Life Index. Recuperado el 19 de abril de 2017, de http://www .oecdbetterlifeindex.org/es/topics/safety-es/

Osborne, J. W. \& Overbay, A. (2004). The power of outliers (and why researchers should always check for them). Practical Assessment, Research \& Evaluation, 9(6), 34-48.

Paoline, E. \& Terrill, W. (2014). Police Culture. Adapting to the Strains of the Job. Durham: Carolina Academic Press. 
Purpura, P. P. (2001). Police and community: Concepts and cases. Boston: Allyn and Bacon.

Rico, J. M. \& Chinchilla, L. (2002). Seguridad ciudadana en América Latina. México: Siglo XXI.

Rivas, L. A. (2006). ¿Cómo hacer una tesis de maestría? México: IPN.

Roberts, D. J. (2006). Law enforcement tech guide for creating performance measures that work: a guide for executives and managers. Washington, D. C.: US Department of Justice, Office of Community Oriented Policing Services.

Salamanca, F. (2004). Análisis comparativo de sistemas de indicadores pertinentes a la relación entre policía y comunidad. Centro de Estudios en Seguridad Ciudadana. Recuperado el 19 de abril de 2017, de http://www.cesc.uchile.cl/publicaciones/op_03_salamanca.pdf

Salvatore, D. (1994). Microeconomics. Nueva York: HarperCollins.

Savelsberg, H. W. (1994). Der ProzeB polizeilicher Entscheidungsfindung. Ein Beitrag zur Soziologie. Hessen: Springer Fachmedien Wiesebaden.

Secretaría de Seguridad Pública Federal. (2010). Nuevo Modelo de Policía. México: ssp.

Servera-Muntaner, J. (2006). Ética policial. Valencia, España: Alfa Delta.

SESNSP. (2011a). Respuesta a las solicitudes de información, folios 2210300028613, 713, y 813, de fecha 12 de agosto de 2013. sesnsp, Unidad de Enlace. México: SESNSP.

SESNSP. (2011b). Encuesta Institucional Sobre Seguridad Pública. México. Recuperado el 30 de junio de 2013, de http://www.secretariadoejecutivo.gob.mx/work/models/Secretariado Ejecutivo/Resource/1105/Informe_Nacional_Encuesta_Institucional_2011.pdf

SESNSP. (2011c). Acuerdo Nacional para la Seguridad, la Justicia y la Legalidad. Recuperado el 30 de junio de 2013, de http:/www.secretariadoejecutivosnsp.gob.mx/work/models/ SecretariadoEjecutivo/Resource/493/ANSJL_18_GOSTO_2011.pdf

SESNSP. (2011d). Sueldos de policías estatales y municipales. Recuperado el 30 del junio de 2013, de http://www.secretariadoejecutivosnsp.gob.mx/work/models/SecretariadoEjecutivo/Resource 1347/1/images/Salarios_de_Policias_2010_y_2011_corregida.pdf

Shane, J. (2007). What every chief executive should know: using data to measure police performance. Nueva York: Looseleaf Law Publications.

Silverman, E. B. (2002). El programa compStat y la policía de distrito en Nueva York. Revista Catalana de Seguretat Pública, (10), 41-46. 
Thompson, J. D. (1967). Organizations in action: Social science bases of administrative theory. New Brunswick: Transaction Publishers.

Tilley, N. (2008). Modern approaches to policing: community, problema oriened and intelligence-led. En Newburn, T. (Ed.). Handbook of policing (pp. 373-403). Cullompton, Portland: Willan Publishing.

Tsai, K. H. \& Huang, C. T. (2012). Technology Synergy, Product Characteristics, and New Product Performance: A Meta-Analytic Review. Canadian Journal of Administrative Sciences/ Revue Canadienne des Sciences de l'Administration, 29(4), 336-347.

Tyler, T. R. (2011). Trust and legitimacy: Policing in the USA and Europe. European Journal of Criminology, (8), 254-266.

Tyler, T. R. \& Huo, Y. (2002). Trust in the law: Encouraging public cooperation with the police and courts. Nueva York: Russell Sage Foundation Publications.

Vallejo, Martos, M. C. (2008). Coraje: La respuesta cultural a los problemas evolutivos de la empresa familiar. Dirección y Organización, (36), 51-60.

Wasserman, R. \& Moore, M. H. (1988). Values in Policing. Perspectives in Policing, (8). Washington, D. C.: U. S. Department of Justice, National Institute of Justice. Recuperado el 19 de abril de 2017, de https://www.ncjrs.gov/App/publications/abstract.aspx?ID=114216

Wells, E. L. \& Falcone, D. N. (2005). Policing in the United States: Developing a Comprehensive Empirical Model. Washington, D. C.: U. S. Department of Justice, National Institute of Justice, NCJ. Recuperado el 19 de abril de 2017, de https://www.ncjrs.gov/app/publications /abstract.aspx? ID $=210830$

Wilson, J. M. (2005). Determinants of Community Policing: An Open Systems Model of Implementation. Washington: U. S.: Department of Justice, National Institute of Justice, NCJ. Recuperado el 19 de abril de 2017, de https:/www.ncjrs.gov/app/abstractdb/AbstractDB Details.aspx?id=233441

Woodward, J. (1958). Management and technology. Londres: Her Majesty's Stationery Office. 